\title{
Energy absorption of safety nets in building construction
}

\author{
E. G. Segovia Eulogio \& R. Irles Más \\ Department of Civil Engineering, University of Alicante, Spain
}

\begin{abstract}
Safety nets used in buildings under construction in Europe are examples of structures under impact. It would be of great interest to evaluate the energy absorbed by the elements making up these devices (nets and supports) in order to improve them and quantify injuries to victims of falls. Experimental tests on type "V" safety nets (EN 1263-1, 1997) were numerically modelled in this work (consisting of dropping a $100 \mathrm{~kg}$ weight from $7 \mathrm{~m}$ above the nets). The model is dynamic and highly non-linear (variable contact, geometric and material nonlinearities). Two adjustment parameters were used to calibrate the numerical model: the equivalent elastic modulus of the net and the equivalent structural damping of the net. A value for each one was found, thus obtaining the same weight drop and maximum strain on the gauges in the experimental tests. The main conclusion, when numerical simulations were carried out using the calibrated model, is the great difference between the energy absorbed by the net and by the supports (ratio: 8-1). This means that, with research into net materials focusing on this impact problem, absorbed energy is an important factor.

Keywords: safety nets, building under construction, structure under impact, absorbed energy, numerical model.
\end{abstract}

\section{Introduction}

In the second half of the $20^{\text {th }}$ century, different safety net systems for buildings under construction were developed in several countries to prevent falls with the consequent risk of serious injury or death.

With the vertical safety net system, practically exclusive to Spain, there have been several accidents involving death or serious injury that have led to them being improved (Saiz et al. [1]). 
Current criteria used to design the anti-fall systems used in the construction of buildings in Europe and subject to impact are based on experimental tests carried out on said systems. If it were possible to obtain a numerical model on which to base these criteria, the cost of verifying the aptitude of said systems would be reduced, with the consequent enormous increase in the number of antifall systems that could be tested numerically. Therefore, it would be of great technical interest if we could obtain a calibrated model on which to base the design criteria of these anti-fall systems, more specifically the vertical type $\mathrm{V}$ safety nets as per EN 1263-1.

This standard states that an anti-fall system consisting of type $\mathrm{V}$ vertical safety nets is apt if, on carrying out certain experimental tests basically consisting of dropping a $100 \mathrm{~kg}$ weight onto the nets from a height of $7 \mathrm{~m}$, no breakage of either the supports or nets is observed, although they may suffer considerable plastic deformation.

The authors of this study have developed a calibrated numerical model of said experimental tests (Fig. 1) on which to base the design criteria and, when carrying out numerical simulations with said model, have extracted all the results of the variables affected for each one. The most interesting results are those referring to absorbed energies and impact factors, key factors in the design and use of these systems.

At this Congress, we present the conclusions referring to the energies absorbed by the different elements making up the device. The importance of these conclusions stems from the fact that it is an energy absorption problem: it is necessary to absorb energy as gently as possible to ensure that the accident victim does not suffer significant injury.

\section{Numerical model}

\subsection{Experimental tests}

Instrument-based experimental tests were carried out using dynamic strain gauge equipment to record the deformation according to time at several points on the steel supports (Irles et al. [2]). A graduated ruler was also used to record, by means of video recordings, the sagging of the net when the weight hit it. The results used to calibrate the model were the maximum deformation recorded on the gauges used and the maximum net sag. The aim was to create a calibrated numerical model that would obtain the same maximum support deformation and maximum net sag as with the instrument-based experimental tests.

\subsection{Characteristics}

As well as having to carry out transient dynamic analysis in order to evaluate the maximum deformation values for the supports and net, the phenomenon in question is highly non-lineal, as:

- Significant movements are generated in the supports and the net (geometric non-linearity), with even the net registering deformation values that cannot be regarded as small. 


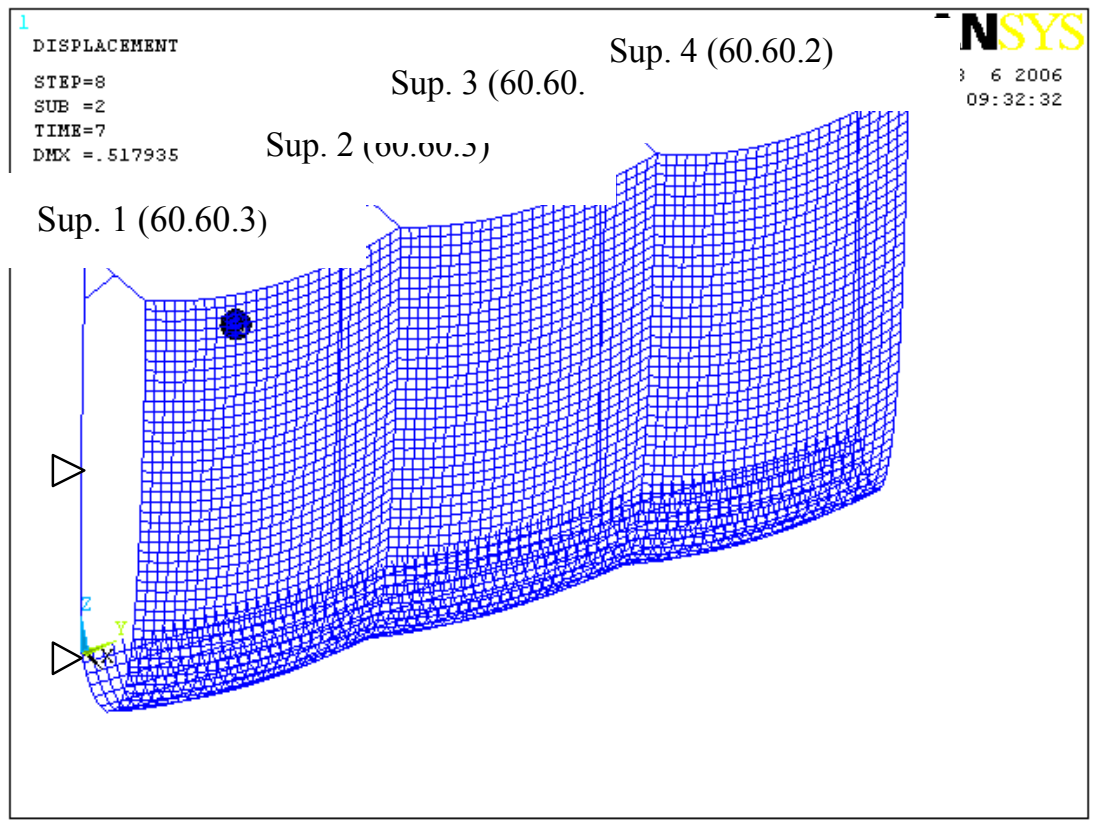

Figure 1: Numerical model showing the moment immediately prior to releasing the weight.

- There is variable contact between the net and the weight (geometric nonlinearity)

- The elastoplastic behaviour of the materials (mechanical non-linearity), modelling the steel as bilinear (yield stress: $235.2 \mathrm{MPa}$; elastic modulus: 205.8 $\mathrm{GPa}$ ), and the net material in a more complex way, as the modelling includes the energy absorption.

- The fact that initial transversal rigidity (Figure 1) is nil, making it necessary to use tensional rigidity in the model (geometric non-linearity).

The model was obtained by using the structural module of the Ansys version 7.1 finite elements package (2003, Swanson Analysis Systems), which modelled the above-mentioned characteristics very efficiently.

\subsection{Modelling the net}

Although the net behaves in a rather complex manner (Paureau et al. [3]), the law of simplified behaviour of the net includes, in an "equivalent" manner, the energy absorption capacity. This equivalence is adjusted according to the experimental results. The proposed model is based on prismatic finite elements with a law of linear behaviour, no resistance to pressure and structural damping.

Two net parameters were used to ensure that the net in the model behaved in the same way as in the experimental tests: 
$\mathrm{k}_{\mathrm{r}}$ : The slope of the straight section of the law of behaviour corresponding to traction.

- $\beta$ : A multiplier of the rigidity matrix to obtain the damping matrix (structural damping).

As will be seen, the values for the two calibration parameters obtained were used in our model to obtain similar results to those obtained in the experimental tests.

\subsection{Control variables}

The results of the model chosen to have similar results to those obtained from the experimental tests and calibrate the model and called control variables were:

- $h$ : Maximum descent undergone by the net $\left(\mathrm{h}_{\mathrm{MOD}}\right.$ and $\mathrm{h}_{\mathrm{EXP}}$, numerical model and experimental test, respectively).

- $\varepsilon_{\mathrm{S}}$ : Maximum deformation measured in one channel of the experimental tests (most significant component, $\varepsilon_{\mathrm{S}, \mathrm{MOD}}$ and $\varepsilon_{\mathrm{S}, \mathrm{EXP}}$, numerical model and experimental test, respectively).

The choice of these parameters and fit variables allows us (Segovia [4]) to believe that the process can be calibrated.

\subsection{Hypothesis}

The hypotheses used to create the model are commonly accepted in the Strength of Materials and were compatible with the results obtained from the experimental tests:

- Small deformations, although with major movement of the steel.

- The laws of the behaviour of materials, which are acceptable given the fact that the numerical model includes the behaviour in an equivalent manner.

- The steel behaves in a homogeneous, continuous and isotropic manner.

-The prismatic elements modelling the supports behave in accordance with Navier's hypothesis and do not influence in the plastification of the shearing stress, a plastic hinge without denting is formed and they undergo uniform stress.

\subsection{Calibration of the model}

For the calibration, the following was defined:

$$
\begin{aligned}
& e_{r}\left(k_{r}, \beta\right)=\frac{h_{M O D}\left(k_{r}, \beta\right)-h_{E X P}}{h_{E X P}} \\
& e_{s}\left(k_{r}, \beta\right)=\frac{\varepsilon_{S, M O D}\left(k_{r}, \beta\right)-\varepsilon_{S, E X P}}{\varepsilon_{S, E X P}}
\end{aligned}
$$

and these functions were estimated by minimum square adjustment after carrying out multiple simulations while varying $\mathrm{k}_{\mathrm{r}}$ and $\beta$.

On resolving the system of equations, the calibration became: 


$$
\left\{\begin{array}{l}
e_{r}\left(k_{r}, \beta\right)=0 \\
e_{s}\left(k_{r}, \beta\right)=0
\end{array}\right.
$$

the solutions of which were the values of the parameters $\mathrm{k}_{\mathrm{r}}$ and $\beta$ that calibrated the model $\left(\mathrm{k}_{\mathrm{r}}=61 \mathrm{daN}\right.$ and $\beta=34 \%$ of the critical value for a frequency of 1 $\mathrm{Hz}$ ). The high structural damping value is due to the fact that the model uses this parameter to simulate all the dissipation of energy in the net, including that due to the tightening of the knots - which is very high in the first few moments.

These adjustment parameters were used to carry out a simulation with the numerical model and it was seen that the values of the variables of the model were similar to those of the experimental tests (with a difference of less than $5 \%$, a permissible error if we bear in mind that other authors (Nicot et al. [5]) regard as acceptable much greater differences in numerical models of similar phenomena).

The high structural damping value is admissible as the model reproduces the maximum valuables of the variables of the phenomenon, leaving the creation of a calibrated model in which the structural damping value reduces over time for later research, as the tightening of the knots only takes place in these first moments of contact.

\section{Energy absorption}

\subsection{The weight}

With regard to the weight, as it descends, the gravitational potential energy is converted into kinetic energy up to the point when it impacts with the net, starts slowing down and transferring the energy to the net, through the work of the weight-net contact forces. The graph corresponding to the evolution of the weight energies in the first three seconds from the weight being released is:

The energy balance corresponding to Figure 2 is:

$$
\nabla \mathrm{E} p w=\Delta E c w+E t n
$$

where:

$-\nabla E p w$ is the reduction in the gravitational potential energy of the weight.

$-\Delta E c w$ is the increase in the kinetic energy of the weight.

- Etn is the energy transferred to the net through the work of the weight-net contact forces.

Figure 2 shows that the total energy to be absorbed is approximately $7.7 \mathrm{~kJ}$ (the potential corresponding to a mass of $100 \mathrm{~kg}$ falling from $7.9 \mathrm{~m}$ - initially 7 $\mathrm{m}$ above the net plus $0.9 \mathrm{~m}$ due to the plastic deformation of the device). 


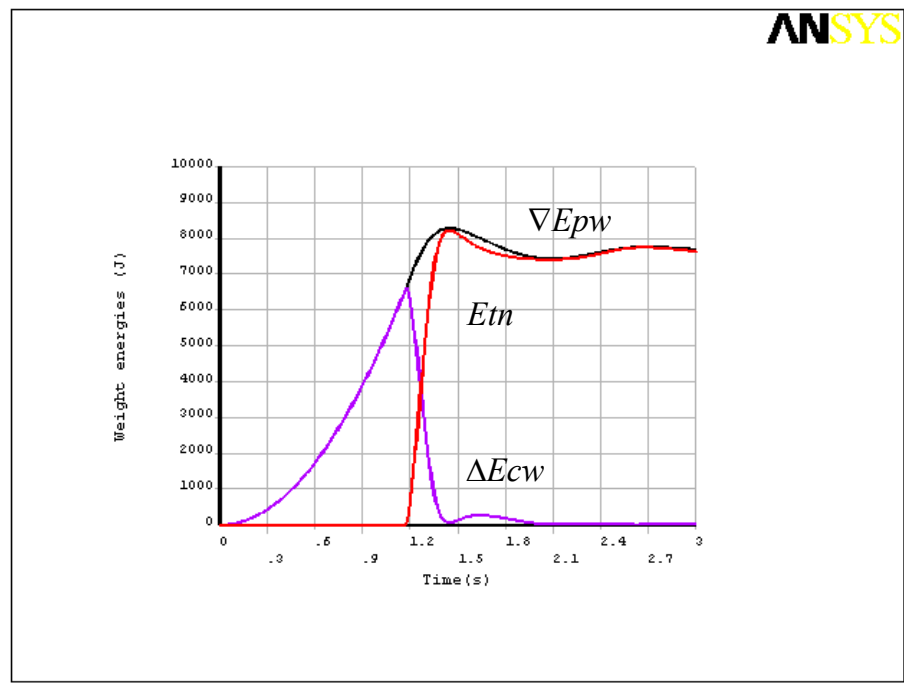

Figure 2: Weight energies.

\subsection{The net}

The energy transferred to the net and the reduction in its potential energy is invested in increasing its kinetic energy, deforming the net (elastic plus plastic), transferring part to the supports and dissipating most throughout the net mainly as a result of knot tightening, as well as plastic deformations. Figure 3 shows that most of the energy that must be absorbed $(7.7 \mathrm{~kJ})$ is done so in the form of dissipation through damping in the model $(6.8 \mathrm{~kJ})$ and much less so by the supports $(0.77 \mathrm{~kJ})$.

Figure 3 shows the evolution of the energy/works according to time in the first three seconds from the weight being released.

The energy balance corresponding to Figure 3 is:

$$
E f w+\nabla \mathrm{E} p n=E d n+W s n+E t s+\Delta E c n
$$

where:

- Efw is the energy proceeding from the weight through the work of the weight-net contact forces.

- $\nabla E p n$ is the reduction in the gravitational potential energy of the net.

- Edn is the energy dissipated by net damping.

- WSn is the deformation work (elastic plus plastic) developed on the net.

- Ets is the energy transferred to the supports by the work of the internal forces that appear between the net and the metal supports.

$-\Delta E c n$ is the increase in the kinetic energy in the net. 


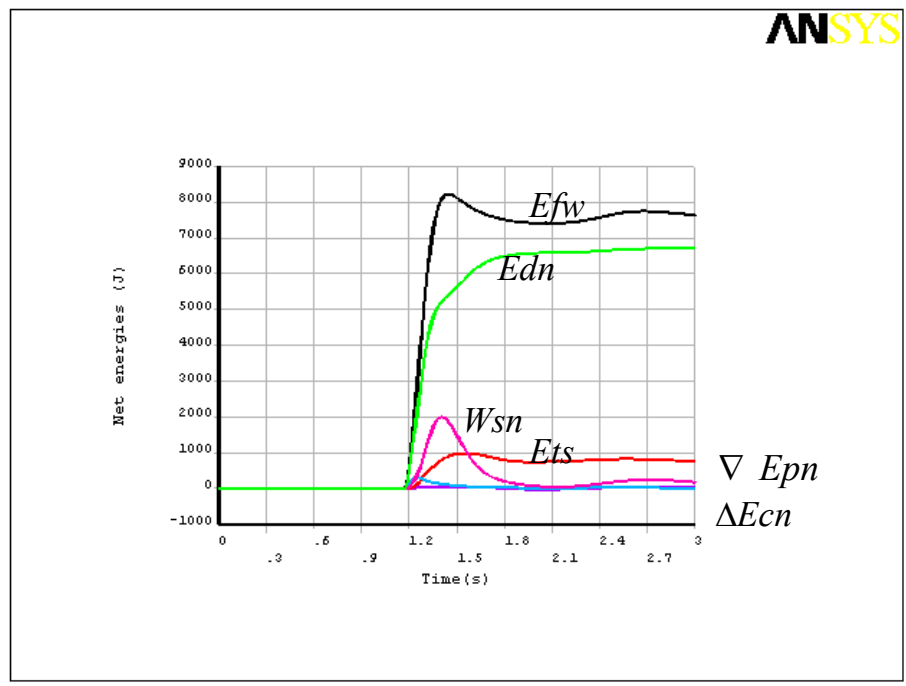

Figure 3: $\quad$ Net energies.

\subsection{The supports}

The energy transmitted from the net to the supports, along with the reduction in their gravitational potential energy is invested in deformation work (elastic plus plastic) and energy dissipated by damping in the supports and the increased kinetic energy of the supports.

It should be pointed out that the energy dissipated by damping in the supports is negligible compared with the deformation work, as a typical structural damping value of $1 \%$ for the $1 \mathrm{~Hz}$. Frequency has been taken and both have been included in a single curve.

Figure 4 shows the evolution of the energy/work of the supports according to time in the first three seconds from the weight being released:

The energy balance corresponding to Figure 4 is:

$$
E f n+\nabla \mathrm{E} p s=W s s+\Delta E c s
$$

where:

- $E f n$ is the energy proceeding from the net through the work of the internal supports-net forces.

- $\nabla E p s$ is the reduction in gravitational potential energy of the supports.

-WSS is the deformation work (elastic plus plastic) developed on the supports plus the energy dissipated by support damping.

- $\Delta E c s$ is the increase in kinetic energy in the supports. 


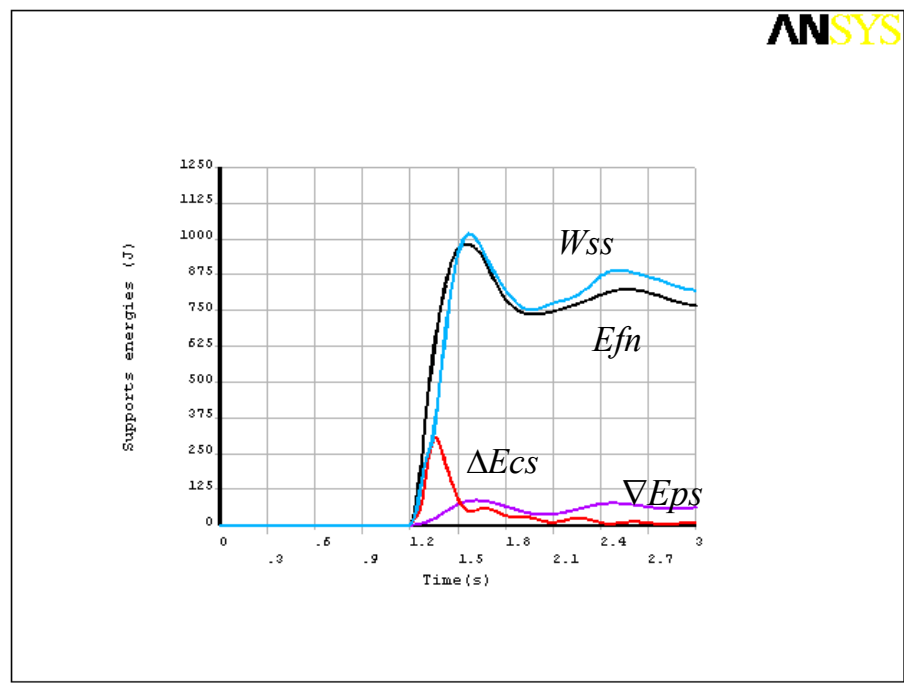

Figure 4: Support energy.

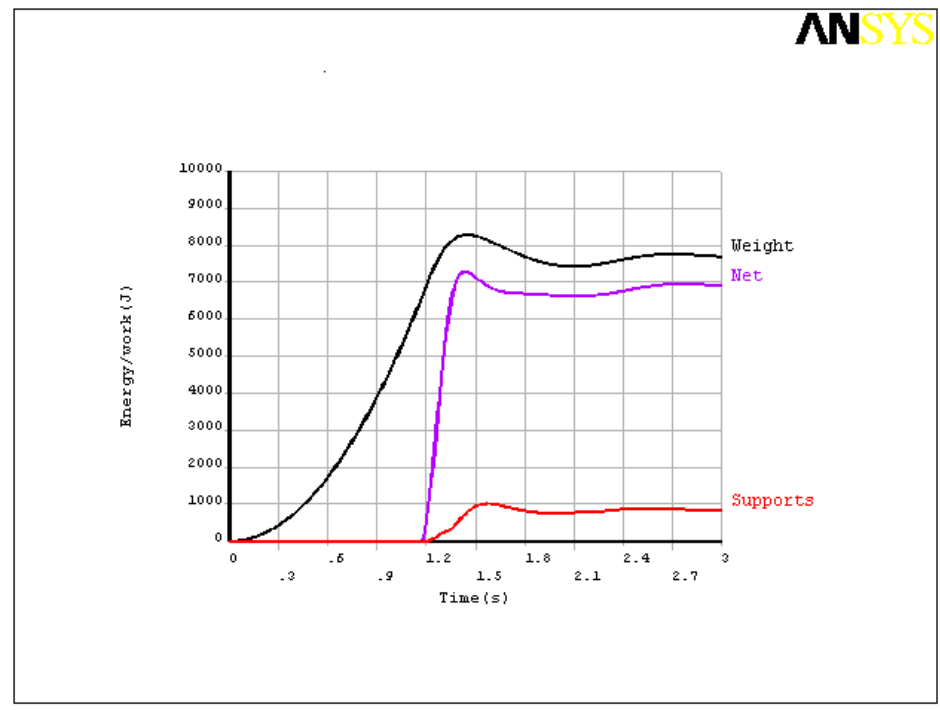

Figure 5: $\quad$ Energy summary.

\section{Conclusions}

Figure 5 summarises the most significant energies involved in the phenomenon. It can be seen that the energy proceeding from the weight that must be absorbed ( $7.7 \mathrm{~kJ}$ - reduction in gravitational potential energy) is largely absorbed by the 
net ( $7 \mathrm{~kJ}$ - damping plus deformation work) and to a much lesser degree by the supports $(0.83 \mathrm{~kJ}$ - deformation work). The energy balance adds up when we add the reductions in the potential energies of the supports and net.

It is clear that with these devices the absorption of energy by the material of which the net is made and its knotting system is a key factor in the efficiency of the system (absorbing energy gently) and the authors of this study feel that further research should be carried out to this end.

\section{References}

[1] Saiz, J., Irles, R., Arcenegui, G.A., Naharro, M., Las Redes de Seguridad en la Construcción., Universidad de Alicante, pages 67-76, 1997.

[2] Irles, R., Maciá, A., Segovia, E., Saiz,. J., Redes verticales de seguridad. Resultados teóricos y experimentales. Proc. of the III European Forum in Science, Security and Health, Universidad de Oviedo, pages 561-564, 1998.

[3] Paureau, J., Parisot, E., Schuler, B., Filets en grandes nappes. Eficacité, performances, règles de pose Cahiers de Notes Documentaires, INRS, 137, France, pages 655-675, 1989.

[4] Segovia, E., G., 4.2.2. Red. Parámetros y variables para la calibración, Criterios para el diseño de los sistemas anticaída utilizados en construcción y sometidos a impacto, Tesis Doctoral, Universidad Politécnica de Valencia, pages 56-61, 2005.

[5] Nicot, F., Cambou, B., Mazzoleni, G., From a constitutive modelling of metallic rings to the design of rockfall restraining nets, Int. J. For num. and an. Meth. In Geomech., 25(1), pp. 49-70, 2001. 